\title{
Chloraea riojana (Chloraeinae-Orchidaceae), una nueva ORQUÍDEA ARGENTINA
}

\author{
ANA SOBRAL ${ }^{1}$ y PATRICIO NOVOA ${ }^{2}$
}

\begin{abstract}
Summary: Chloraea riojana (Chloraeinae-Orchidaceae), a new orchid of Argentina. A new species of orchid in the genus Chloraea, sect. Foliosae, is described. This species native from La Rioja (Argentina) represents the southernmost limit of distribution of this section, which ranges from Southwestern Peru to Northwestern Argentina. This new taxon is based on significant morphological and phenological differences, together with its geographical isolation relative to the remaining species within the genus Chloraea. The most obvious difference with the most related species (Chloraea praecincta Speg. et Kranzl. and Chloraea reticulata Schlechter) is the color of the labellar lamina, which is deep yellow in the new species and white in the other two. Among other differences $C$. praecincta do not have nectar pits and $C$. reticulata has the widened base of the column.
\end{abstract}

Key words: Chloraea, La Rioja, native flora, new species, Northwestern Argentina, taxonomy, terrestrial orchid.

\begin{abstract}
Resumen: Se describe una nueva especie de orquídea del género Chloraea, secc. Foliosae. Encontrada en provincia de La Rioja, representa el nuevo límite sur de distribución de la sección, presente desde el sudoeste de Perú hasta el noroeste de Argentina. Las diferencias significativas, morfológicas (de color del perigonio), fenológicas y la separación geográfica con las otras especies del género justifican la creación de este nuevo taxón. La diferencia más evidente con las especies afines (Chloraea praecincta Speg. et Kranzl. y Chloraea reticulata Schlechter) es el color de la lámina del labelo, amarillo intenso en la especie riojana y blanco en las dos afines. Asi mismo, presenta fosas nectaríferas (C. praecincta no tiene) y la columna es recta (la de C. reticulata tiene la base ensanchada).
\end{abstract}

Palabras clave: Chloraea, flora nativa, La Rioja, noroeste argentino, nueva especie, orquídea terrestre, taxonomía.

El género Chloraea Lindl. (Orchidaceae, Subtribu Chloraeinae), (Chase et al., 2003) es endémico de Sudamérica (Dressler, 1993) y comprende 55 especies terrestres (Govaerts, 2013), de las cuales 24 habitan en Argentina (Zuloaga et al., 2008). Fue propuesto por Lindley (1827) basado en láminas de Feuillée (1714) y en material coleccionado por Mc Rae (sin publicar). Kraenzlin (1904) propuso su división en cuatro secciones: 1. Uniflorae, con cuatro especies en Argentina, Brasil y Uruguay, que actualmente pertenecen al género Geoblasta Barb. Rodr., Correa (1969)" por la siguiente: "que fueron posteriormente

\footnotetext{
${ }^{1}$ Instituto Multidisciplinario de Biología Vegetal, Universidad Nacional de Córdoba, CONICET. Av Vélez Sarsfield 299, CP: 5000, Córdoba, Argentina, anacsobral@gmail.com

2 Jardín Botánico Nacional, Camino El Olivar 305, El Salto, Viña del Mar, Chile.
}

transferidas al género Geoblasta Barb. Rodr., Correa (1969), que es actualmente sinónimo de Bipinnula Comm. ex Juss. (1789), (Cisternas et al., 2012a); 2. Lamellatae, con el labelo recorrido por laminillas; 3. Papillosae, con el labelo cubierto por papilas y el perigonio reticulado; 4. Euchloraea, con papilas atenuadas y perigonio no reticulado. Posteriormente, Hauman (1922), criticó las monografías de Kraenzlin y de Reiche (1910) y estableció que las casi 80 especies conocidas hasta el momento se distribuían en tres áreas completamente independientes y separadas entre si, denominándolas: I. Área PatagónicoChilena, con 60 especies aproximadamente; II. Área Sudbrasilo-Platense con 3 a 4 especies y III. Área Tucumano-Boliviana con 12 especies (Fig. 1) y observó que todas las especies del área TucumanoBoliviana se distinguen por su tallo hojoso en toda su extensión, característica compartida únicamente 


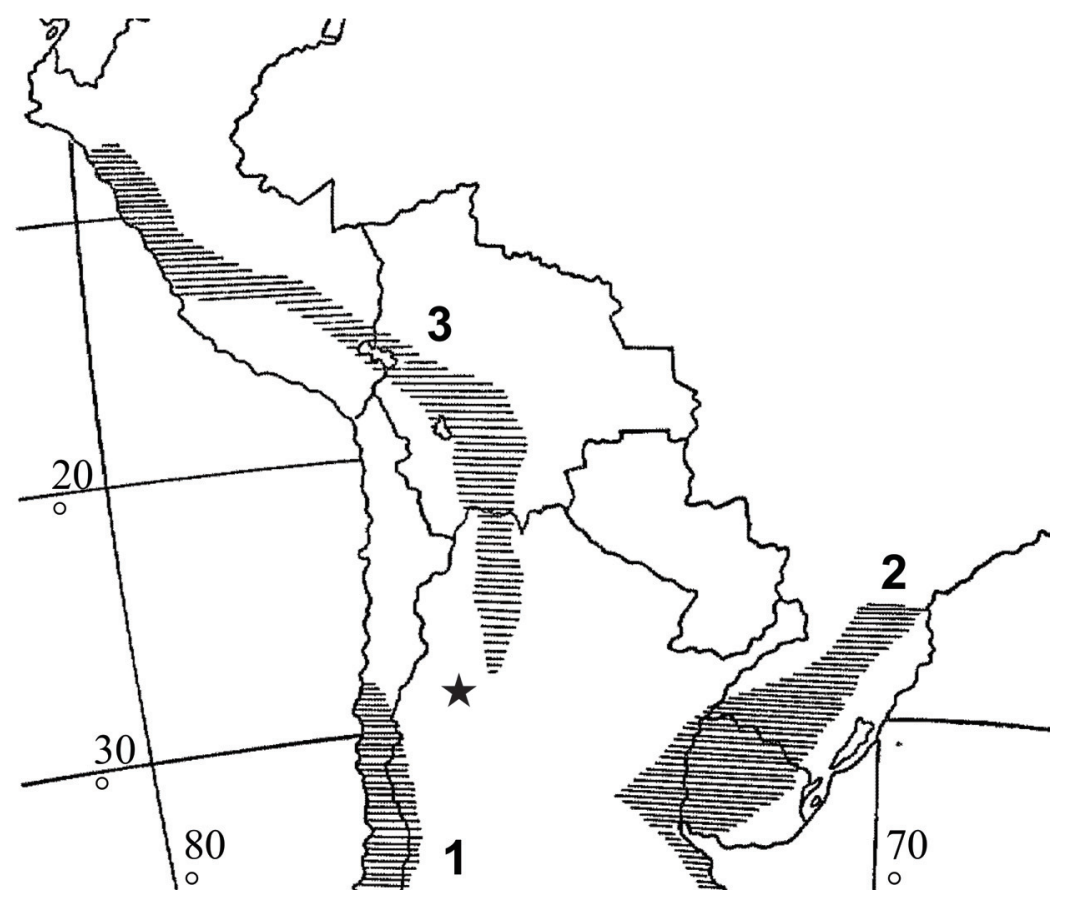

Fig. 1. Distribución del género Chloraea (Orchidaceae) en 3 grupos disjuntos, sensu Hauman (1922): 1. Área I Patagónica-Chilena, 2. Área II Sudbrasilo-Platense y 3. Área III Tucumano-Boliviana. La estrella señala la localización de C. riojana en la Sierra de Velasco, La Rioja, Argentina (adaptado de Correa, 1969).

por la especie Chloraea cylindrostachya del área I, Patagónico-Chilena, mientras que las demás poseen una roseta de hojas radicales y escapos sin hojas. Consecuentemente propone la división del género en: 1 Uniflorae, con una a dos especies SudbrasiloPlatenses, y 2 Spiciflorae, que divide a su vez en A. Rosulatae, con hojas en roseta radical, y B. Foliosae, con escapos hojosos. Luego Correa (1969) reconoce la división geográfica de Hauman y cambia los nombres por: a. Grupo Oriental, correspondiente al grupo II, Sudbrasilo-Platense, b. Grupo Septentrional, correspondiente al grupo II, Tucumano-Boliviano y c. Grupo Occidental, correspondiente al gupo I, Patagónico-Chileno, sin justificar este cambio de nombres superfluo.

Según análisis filogenéticos recientes (Chemisquy \& Morrone, 2010 y Cisternas et al., 2012) el género Chloraea no es monofilético, al igual que ninguno de los que componen la subtribu Chloraeinae. Chloraea chica queda incluida en el género Gavilea, y varias otras especies, entre ellas C. cylindrostachya y $C$. reticulata son basales y se encuentran separadas del grupo principal de Chloraea por el clado que componen las especies de Gavilea y Bipinnula.

El objetivo de este trabajo es describir una especie de orquídea del género Chloraea, recientemente descubierta en la Provincia de La Rioja (Dpto. Castro Barros), Argentina. Las características morfológicas propias, de coloración del perigonio, y fenológicas, la diferencian de los miembros afines del género (Tabla 1). Adicionalmente presenta con estas una discontinuidad geográfica y su ubicación amplia el límite meridional del área III Tucumano-Boliviana de distribución del género y de la sección Foliosae.

\section{Chloraea riojana Sobral A. \& Novoa P., sp. nov.}

Herba erecta, $50 \mathrm{~cm}$ alta; tota altitudine foliata; sepalo dorsali lanceolato, sepalis lateralibus lanceolatis, acutis, sepalis et petalis albescentibus viridi-reticulatis; labello luteo, sub-trilobo ipsa base cristis 7-9 elevatis, apicem versus in papillis dissolutis, margine arcte undulata, antice acuto vel obtuso, incrassato.

TIPO: Argentina. Prov. La Rioja, Dpto. Castro Barros: Quebrada de Anillaco, 18-XI-2011, A. 


\section{A. Sobral y P. Novoa - Chloraea riojana, una nueva orquídea argentina}

Tabla 1. Cuadro comparativo de las diferencias morfológicas más significativas entre $C$. riojana y las especies más afines: Chloraea praecincta y Chloraea reticulata.

\begin{tabular}{|c|c|c|c|}
\hline Atributo & C. riojana & C. praecincta & C. reticulata \\
\hline Labelo & $\begin{array}{l}\text { Panduriforme a escasamente } \\
\text { trilobado }(11-13 \times 6-8 \mathrm{~mm})\end{array}$ & $\begin{array}{l}\text { Entero, panduriforme a } \\
\text { oscuramente trilobado } \\
(11-16 \times 7-12 \mathrm{~mm})\end{array}$ & $\begin{array}{l}\text { Generalmente trilobado } \\
(13-18 \times 7-12 \mathrm{~mm})\end{array}$ \\
\hline Ápice del labelo & $\begin{array}{l}\text { Agudo a obtuso, } \\
\text { engrosado, recurvo }\end{array}$ & $\begin{array}{l}\text { Muy ondulado, ancho, } \\
\text { carnoso, reflejo }\end{array}$ & $\begin{array}{l}\text { Redondeado, ancho, } \\
\text { obtuso, carnoso, reflejo }\end{array}$ \\
\hline Base del labelo & Ancha & Angostada & Angostada \\
\hline $\begin{array}{l}\text { Apéndices de la } \\
\text { lámina del labelo }\end{array}$ & $\begin{array}{l}\text { Laminillas falcadas y papilas } \\
\text { gruesas capitadas y coloreadas }\end{array}$ & $\begin{array}{l}\text { Filiformes rectos, abundantes } \\
\text { verrugas y laminillas }\end{array}$ & $\begin{array}{l}\text { Papilas gruesas con } \\
\text { ápice verde oscuro }\end{array}$ \\
\hline $\begin{array}{l}\text { Color de la } \\
\text { lamina del labelo }\end{array}$ & Amarillo intenso & Blanco & Blanco-crema \\
\hline Sépalo dorsal & $\begin{array}{l}\text { Lanceolado, agudo } \\
(18-20 \times 4-5 \mathrm{~mm})\end{array}$ & $\begin{array}{l}\text { Oblongo agudo a subobtuso } \\
(15-25 \times 4,5-6 \mathrm{~mm})\end{array}$ & $\begin{array}{l}\text { Lanceolado, agudo, aquillado } \\
(18-25 \times 6-8 \mathrm{~mm})\end{array}$ \\
\hline Sépalos laterales & $\begin{array}{l}\text { Lanceolado, oblicuos, } \\
\text { asimétricos }(15-18 \times 3-5 \mathrm{~mm})\end{array}$ & $\begin{array}{l}\text { Ensiformes, falcados agudos } \\
(14-20 \times 3,5-5 \mathrm{~mm})\end{array}$ & $\begin{array}{l}\text { Lanceolados, agudos, oblicuos } \\
\text { asimétricos (15-23 x 4-9 mm) }\end{array}$ \\
\hline Pétalos & $\begin{array}{l}\text { Lanceolados, subagudos, } \\
\text { ovados, algo cóncavos, } \\
\text { levemente asimétricos } \\
(13-15 \times 5-7 \mathrm{~mm})\end{array}$ & $\begin{array}{l}\text { Lanceolados, subagudos } \\
(14,5-20 \times 5-8 \mathrm{~mm})\end{array}$ & $\begin{array}{l}\text { Ovados, subagudos a } \\
\text { obtusos, algo cóncavos } \\
(15-20 \times 8-11 \mathrm{~mm})\end{array}$ \\
\hline Unguícula & Ausente & Cóncava & Cóncava \\
\hline Columna & Recta, rojo intenso en la base & $\begin{array}{l}\text { Recta, naranja } \\
\text { amarillento en la base }\end{array}$ & $\begin{array}{l}\text { Ensanchada, rojo } \\
\text { intenso en la base }\end{array}$ \\
\hline $\begin{array}{l}\text { Fosas } \\
\text { nectaríferas }\end{array}$ & Dos & Ausentes & Dos \\
\hline Antesis & $\begin{array}{l}\text { Primavera, afila al } \\
\text { momento de la floración }\end{array}$ & $\begin{array}{l}\text { Verano, en presencia } \\
\text { de roseta basal }\end{array}$ & $\begin{array}{l}\text { Otoño, en presencia } \\
\text { de roseta basal }\end{array}$ \\
\hline Distribución & La Rioja & Jujuy, Tucumán y Catamarca & Catamarca, Jujuy, Bolivia, Perú \\
\hline
\end{tabular}

Quinteros 10591 (Holotypus CHAM) (Fig. 2 y 3, Ilustradora: Lena Merie Echelle).

Hierba erecta, perenne, escapo floral $45-55 \mathrm{~cm}$ alt., cubierto de hojas caulinares (Fig. 2A). Raíces fasciculadas, abundantes, aprox. $27 \mathrm{~cm}$ largo, nacen de un rizoma pequeño debajo del tallo (Fig. 2B). Hojas radicales lanceoladas, ápice agudo, 12,5$17,5 \times 4-5,5 \mathrm{~cm}$, formando una roseta senescente durante la antesis. Hojas caulinares lanceoladas, envainadoras, 5-7,5 × 1-5 cm. Inflorescencia 17$22 \mathrm{~cm}$ de largo, 19-21 flores erguidas en racimo de desarrollo acrópeto (Fig. 2C). Ovario $10 \times 3$ $\mathrm{mm}$. Brácteas florales membranáceas, reticuladas, 19-21 × 3-4 mm, cubren y sobrepasan el ovario alcanzando el primer tercio del sépalo dorsal. Flores resupinadas, $3 \mathrm{~cm}$ de alto $\mathrm{x}$ 3,5 $\mathrm{cm}$ de ancho, aroma dulce que recuerda a la vanilla. Sépalos y pétalos membranáceos, blancos con retículo verde oscuro (excepto el labelo). Sépalo dorsal oblongooblanceolado, agudo, simétrico, 18-20 × 4-5 mm.
Sépalos laterales lanceolados, agudos, oblicuos y asimétricos, 15-18 × 3-5 mm. Pétalos subagudos, anco-elíptico a ovados, algo cóncavos y levemente asimétricos, 13-15 × 5-7 mm (Fig. 2D). Labelo panduriforme a escasamente trilobado, 11-13 $\times$ 6-8 $\mathrm{mm}$ en la porción media, de color amarillo intenso (Fig. 2E), borde verde oscuro, ligeramente ondeado y engrosado notoriamente en la mitad anterior, ápice agudo a obtuso y ligeramente recurvo, lámina recorrida por 7 a 9 líneas de laminillas falcadas que hacia delante se deshacen en papilas gruesas de color amarillo con el ápice verde. Columna geniculada, corta en comparación con resto del género, 11-12 × $3 \mathrm{~mm}$ (Fig. 2F); ala recta de color verde muy claro, ensanchada hacia el ápice, cubriendo la parte posterior del ginostemio, base con un par de fosas nectaríferas vivamente coloreadas de rojo; estigma triangular, 3 $\times 4 \mathrm{~mm}$, ocupando menos de un tercio del largo de la columna; rostelo inconspicuo; polinario grueso, de color verde con los bordes amarillos (Fig. 2G); 
Bol. Soc. Argent. Bot. 48 (3-4) 2013

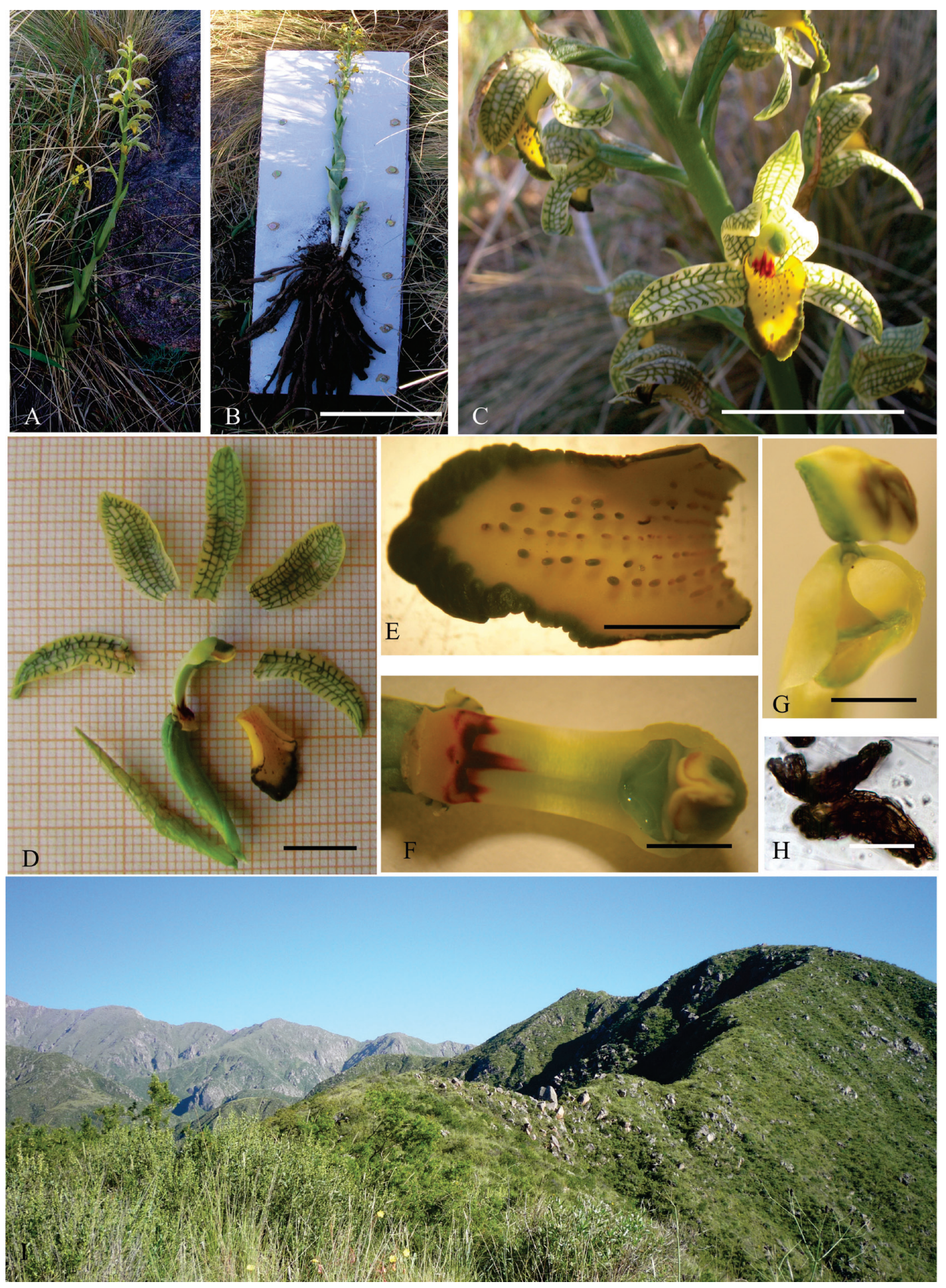

Fig. 2. Chloraea riojana. A: En su hábitat natural. B: Planta completa. C: Inflorescencia D: Piezas florales. E: Labelo. F: Columna. G: Polinario H: Semillas. I: Sitio del hallazgo. (Escalas = B: $20 \mathrm{~cm} ;$ C: $0,20 \mathrm{~cm} ; \mathbf{D}: 1 \mathrm{~cm}$; E: $0,5 \mathrm{~cm} ; \mathrm{F}$ y G: $0,25 \mathrm{~cm} ; \mathrm{H}: 0,02 \mathrm{~cm})$. 


\section{A. Sobral y P. Novoa - Chloraea riojana, una nueva orquídea argentina}
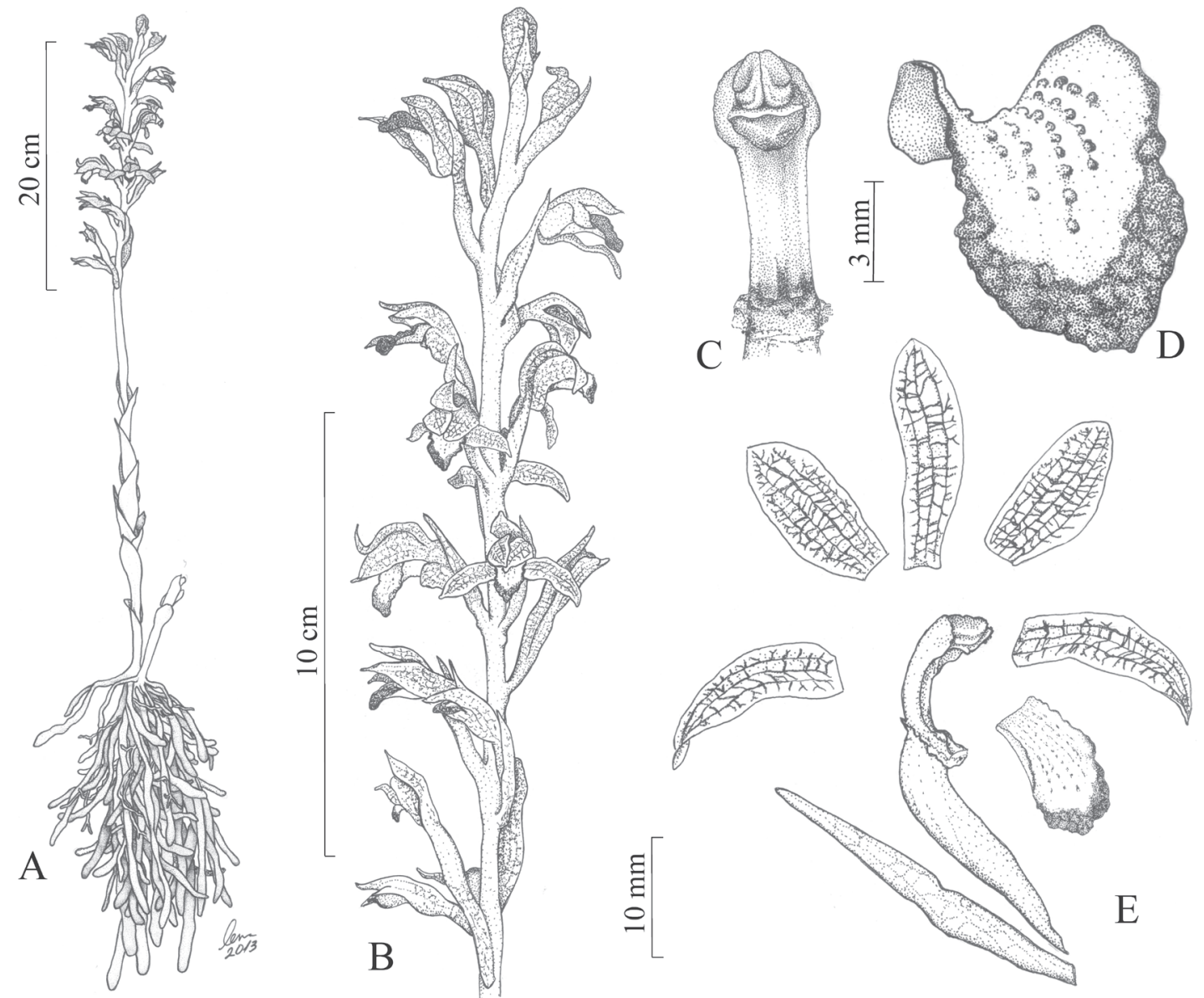

Fig. 3. Chloraea riojana. A: Planta completa. B: Espiga. C: Columna. D: Labelo. E: Piezas florales.

polinios cuatro, dos por teca. Cápsulas $2,5 \times 0,5$ $\mathrm{cm}$. Semillas de $370-400 \times 80-110 \mu \mathrm{m}(\mathrm{N}=35)$ (Fig. 2H).

Fenología: La floración ocurre a mediados de noviembre y la fructificación a principios de diciembre. Durante la antesis floral la roseta radical de hojas se encuentra senescente y reinicia su desarrollo luego de la fructificación.

Distribución y hábitat: Especie nativa de la provincia de La Rioja. Solo conocida en la localidad tipo (Sierra de Velasco, La Rioja).

Las plantas se ubican a lo largo del filo del cerro (Fig. 2I), en un área circunscripta de aproximadamente 2 ha, a más de $2.400 \mathrm{~m}$ de altitud (desde 2.448 m hasta 2.592 m.s.n.m.). La población encontrada asciende a 50-60 individuos creciendo principalmente sobre la ladera occidental, en concavidades del relieve donde se concentra la humedad y el suelo está más desarrollado, también bajo arbustos espinosos, entre rocas o matas de gramíneas, protegidas de los herbívoros.

La región pertenece a la provincia fitogeográfica de la prepuna (Cabrera, 1976) y a la eco-región del monte de sierras y bolsones (Burkart et al., 1999). A menor altitud, en los valles, se conecta con el desierto del monte dominado por Larrea divaricata y en las quebradas más húmedas con el Chaco Serrano. La comunidad vegetal en el sitio es una estepa arbustiva xerófila con afloramientos rocosos.

Los registros de Chloraea más próximos a la localidad tipo de la nueva especie corresponden a C. praecincta Speg. \& Kraenzl., Pomán, 84 $\mathrm{Km}$ en dirección noreste y a $C$. reticulata Schltr., 
Bol. Soc. Argent. Bot. 48 (3-4) 2013

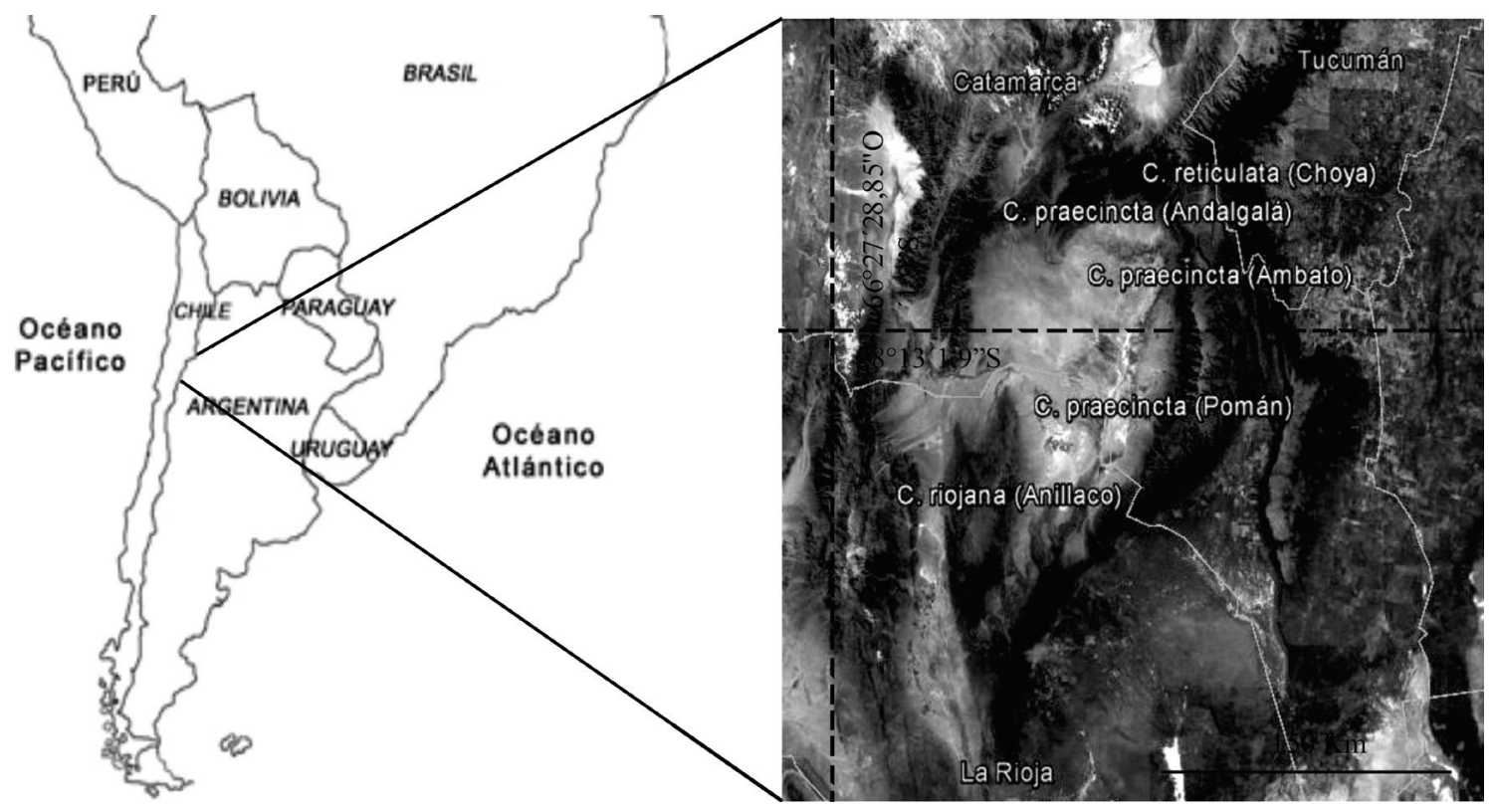

Fig. 4. Localización geográfica del sitio de hallazgo de Chloraea riojana en Anillaco, La Rioja y de las especies afines en la provincia de Catamarca (Chloraea praecincta y Chloraea reticulata). La línea gris señala el límite entre provincias.

Quebrada de Choga, $152 \mathrm{Km}$ hacia el norte, ambas sobre la Sierra de Ambato (Catamarca), (Fig. 4). Amplios valles áridos y la extensa cuenca del salar de Pipanaco (Catamarca y La Rioja) separan estas poblaciones. Los cordones montañosos de Velasco y Ambato se conectan a través de sierras paralelas de distinta altitud y fitogeografía como la sierra de Mazán, el cordón del Carrizal y la sierra de La Punta (La Rioja).

Hacia el sur las poblaciones más cercanas son de Chloraea disoides var. picta (Phil. ex
Kraenzl.) M.N. Correa, en Tunuyán, Mendoza, apartada $565 \mathrm{Km}$. Esta especie pertenece a la secc. Rosulatae y al área I, Patagónico-Chilena. La posición geográfica representa un nuevo límite de distribución meridional del grupo I TucumanoBoliviano formado casi íntegramente por especies de la secc. Foliosae, que habitan desde Catamarca hasta sur de Perú y Bolivia. Adicionalmente dicho punto se encuentra sobre la diagonal árida Argentina, zona donde no hay registros históricos de colectas de orquídeas.

Clave para las especies del grupo septentrional Argentino de Chloraea

1. Flores amarillas o naranjas

2. Labelo trilobulado

3. Pétalos y sépalos amarillos reticulados

3'. Pétalos y sépalos naranjas no reticulados

C. cogniauxii

C. biserialis

2'. Labelo entero a pandurado

4. Labelo recorrido por laminillas 


\section{A. Sobral y P. Novoa - Chloraea riojana, una nueva orquídea argentina}

5. Labelo largamente unguiculado, laminillas devienen en verrugas, planta alta hasta $80 \mathrm{~cm}$ alt.

C. laxiflora

5'. Labelo no unguiculado, laminillas devienen en apéndices unciformes, planta baja hasta $20 \mathrm{~cm}$ alt.

4’. Labelo recorrido por apéndices cilíndricos capitados escasos

C. phoenicea

C. elegans

1'. Flores blancas con retículo verde excepto el labelo

6. Labelo amarillo, borde carnoso verde oscuro

C. riojana

6'. Labelo blanco

7. Labelo trilobulado o profundamente panduriforme

8. Lámina del labelo recorrida por papilas gruesas de ápice verde, labelo trilobado

8' Lámina del labelo desnuda, labelo profundamente panduriforme

C. reticulata

C. subpandurata

7'. Labelo cuneiforme o levemente panduriforme

9. Labelo cuneiforme, recorrido por laminillas enteras bajas

C. castillonii

9' Labelo panduriforme a oscuramente trilobado, recorrido por apéndices rectos filiformes y hoces

C. praecinta

\section{Agradecimientos}

Los autores agradecen al Dr. Juan García Massini, descubridor de esta nueva orquídea, por haberla cedido para su publicación y por su apoyo incondicional con la preparación del manuscrito. A Mauricio Cisternas por las importantes correcciones y comentarios sobre la taxonomía de Chloraeinae. Además, a Antonio Quinteros por su imprescindible colaboración en los trabajos de campo.

\section{Bibliografía}

BURKART, R., N. BÁRBARO, R. SÁNCHEZ \& D. GÓMEZ (eds.). 1999. Eco-regiones de la Argentina. Administración de Parques Nacionales, Programa de Desarrollo Institucional Ambiental, Buenos Aires.

CABRERA, Á. L. 1976. Regiones fitogeográficas argentinas. En: Kugler W.F. (ed.), Enciclopedia argentina de agricultura y jardinería, Tomo 2, fasc. 1, pp. 1-85. 2da edición, Acme, Buenos Aires.

CHASE, M. W, K. M. CAMERON, R. L. BARRET \& J. V. FREUDENSTEIN. 2003. DNA data and Orchidaceae systematics: a new phylogenetic classifications. In: DIXON, K. W., S. P. KELL, R. L.
BARRET \& P. J. CRIBB (eds.), Orchid conservation, pp. 69-89. Natural History Publ., Kota Kinabalu.

CHEMISQUY, M. A. \& O. MORRONE. 2010. Phylogenetic analysis of the subtribe Chloraeinae (Orchidaceae): a preliminary approach based on three chloroplast markers. Aust. Syst. Bot. 23: 38-46.

CISTERNAS, M. A., G. A. SALAZAR \& G. VERDUGO. 2012a. Transfer of Geoblasta Pennicillata to Bipinnula (Chloraeinae, Orchidaceae). Phytotaxa 64: 9-10.

CISTERNAS, M. A., G. A. SALAZAR, G. VERDUGO, P. NOVOA, X. CALDERÓN \& M. A. NEGRITOS. 2012b. Phylogenetic analysis of Chloraeinae (Orchidaceae) based on plastid and nuclear DNA sequences. Bot. J. Linn. Soc. 168: 258-277.

CORREA, M. 1969. Chloraea género sudamericano de Orchidaceae. Darwiniana 15: 374-500.

DRESSLER, R. L. 1993. Phylogeny and classification of the orchid family. Dioscorides Press, Portland.

FEUILLÉE, L. E. 1714-1725. Journal des observations physiques, mathematiques et botaniques. Faites par l'ordre du Roy sur les côtes orientales de l'Amerique meridionale, \& dans les Indies Occidentales. Paris.

GOVAERTS, R. (Compiler). 2003. World Checklist of Orchidaceae, Facilitated by the Royal Botanic Gardens, Kew. http://apps.kew.org/wcsp/ Retrieved 27-02-2013. 
Bol. Soc. Argent. Bot. 48 (3-4) 2013

HAUMAN, L. 1922. La distribución Geográfica del género "Chloraea" Lindl. Physis 5: 293-295.

KRAENZLIN, F. 1904. Orchidacearum genera et species. Exposuit Fritz Kraenzlin. Vol. II, P. 1. Bayer \& Müller, Berlin.

LINDLEY, J. 1827. Remarks upon the Orchidaceous plants of Chile. Quart. J. Sci. Lit. Arts. I.

REICHE, C. 1910. Orchidaceae Chilenses. Ensayo de una monografía de las Orquídeas de Chile. Anales Museo Nac. Chile, Botánica 18: 1-88.
ZULOAGA, F. O., O. MORRONE \& M. BELGRANO (eds.). 2008. Catálogo de las plantas vasculares del Cono Sur. Pteridophyta, Gymnospermae y Monocotyledonae. Vol. 1. Monogr. Syst. Bot. Missouri Bot. Gard. 107.

Recibido el 8 de noviembre de 2012, aceptado el 30 de abril de 2013 . 